\title{
Cultura tributaria como instrumento para una efectiva recaudación de impuestos
}

\section{Tax culture as an instrument for effective tax collection}

\author{
Nalmi Ramos, ${ }^{1}$ Shirly Chero, Wilmer Vidaurre, Max Urbina, ${ }^{4}$ Carmen Rosas ${ }^{5}$
}

\section{RESUMEN}

La presente investigación titulada "CULTURA TRIBUTARIA PARA INCREMENTAR LA RECAUDACIÓN DE IMPUESTOS EN LOS COMERCIANTES DEL MERCADO MOSHOQUEQUE, CHICLAYO", tuvo como objetivo general diseñar una propuesta de Cultura Tributaria para incrementar la Recaudación de impuestos de los comerciantes del mercado Moshoqueque, Chiclayo, se utilizó el tipo de investigación descriptivo - propositivo y se aplicó un diseño no experimental. Como muestra estuvo conformada por 64 comerciantes del mercado Moshoqueque, la técnica utilizada para la recolección de datos fue la encuesta y como instrumento el cuestionario. Entre sus resultados se hallaron que el nivel de cultura tributaria es regular y así como el nivel de recaudación de impuestos. Por lo tanto, se llegó a la conclusión que es de gran importancia la propuesta de crear estrategias de cultura tributara para incrementar la recaudación de impuestos en los comerciantes del mercado Moshoqueque.

Palabras Clave: cultura tributaria, recaudación de impuestos, importancia de impuestos.

\section{ABSTRACT}

The present research work entitled "TAX CULTURE TO INCREASE TAX COLLECTION IN MOSHOQUEQUE MARKET TRADERS, CHICLAYO”, had as its general objective the design of a Tax Culture proposal to increase the tax collection of the producers of the Moshoqueque market, Chiclayo, the descriptive-purposeful type of research was analyzed and a non-experimental design was applied. As a sample it was made up of 64 traders from the Moshoqueque market, the technique used for data collection was the survey and the questionnaire as an instrument. Among its results is the level of regular tax culture and the level of tax collection. Therefore, he concluded that the proposal to create tax culture strategies to increase tax collection in the merchants of the Moshoqueque market is of great importance.

Key Words: tax culture, tax collection, tax importance.

1. Estudiante en Contabilidad, Universidad Señor de Sipán S.A.C., Pimentel - Chiclayo, Perú, rsancheznalmiyo@crece.uss.edu.pe 2. Estudiante en Contabilidad, Universidad Señor de Sipán S.A.C., Pimentel - Chiclayo, Perú, csantistebanshi@crece.uss.edu.pe 3. Doctor en Educación, Maestro en Docencia y Gestión Universitaria, Escuela de Contabilidad, Facultad de Ciencias Empresariales, Universidad Señor de Sipán S.A.C., Pimentel - Chiclayo, Perú, vidaurrewil@crece.uss.edu.pe

4. Vicerrector de Investigación, Innovación y Emprendimiento Universidad Alas Peruanas, Contador Público y Licenciado en Administración egresado de la Universidad Nacional de Trujillo, Maestro en Ciencias Económicas con mención en Finanzas egresado de la Escuela de Posgrado de la Universidad Nacional de Trujillo, Vicerrector de Investigación Universidad Alas Peruanas, urbina10@hotmail.com

5. Doctora en Educación, Magíster en Docencia Universitaria, Maestra en Ciencias Económicas con mención en Tributación Contadora Publica, Licenciada en Administración, Docente Ordinaria de la Universidad Señor de Sipán, categoría principal, celvirarp @crece.uss.edu.pe 


\section{INTRODUCCIÓN}

En la actualidad se ha observado altos índices de informalidad en comerciantes de mercados, mismos que tienen niveles bajos de cultura tributaria, debido a diversos motivos tanto por una falta interés de la entidad recaudadora como por los contribuyentes, perjudicando el nivel de recaudación de impuestos.

Esta investigación nació de la necesidad de aumentar la recaudación tributaria en los comerciantes del mercado Moshoqueque, donde muchos de estos contribuyentes desconocen el proceso para cumplir con las contribuciones tributarias, por tal razón se formuló el siguiente problema ¿De qué manera la propuesta de una cultura tributaria incrementa la recaudación de impuestos en los comerciantes del mercado Moshoqueque, Chiclayo?, en función de lo mencionado se plantea la hipótesis: La propuesta de una Cultura tributaria incrementa la recaudación de impuestos.

Centro de Gestión Tributaria de Chiclayo (CGT, 2018), refiere que, debido a la falta de interés de pago de impuestos por parte de la persona (natural o jurídica), el CGT a través del programa Fomento a la Cultura Tributaria, brindaron charlas educativas sobre gestión tributaria en estudiantes de los últimos grados de la Institución Santa Magdalena Sofía, con el objetivo de que los alumnos conozcan la importancia que generan los tributos en la sociedad.

Los posibles problemas generado en este contexto es la alta informalidad que existente por los comerciantes del mercado Moshoqueque con el fin de evadir impuestos que es el primer factor que influye en los problemas de recaudación de impuestos, la falta de educación tributaria en relación a los cambios constantes que existen en las normas tributarias, la insatisfacción por parte de la persona (natural o jurídica) respecto a los malos manejos del estado referente a los impuestos recaudados generando malestar en los comerciantes y la falta de motivación de la persona (natural o jurídica) en cumplir con sus obligaciones tributarias debido a los altos impuestos y salida de ingreso que afecta su economía. Es por ello que se hace necesario realizar esta investigación que promueva una cultura tributaria en los comerciantes tomando conciencia en que al cumplir fielmente con el pago de tributos estamos ayudando al crecimiento económico de nuestra región mejorando los servicios públicos y por ende la calidad de vida de las personas.

De acuerdo a la investigación de Bermúdez (2016) el autor tuvo como propósito en su investigación mejorar el conocimiento tributario y la cultura tributaria a través del pago de impuestos; aplicando una investigación descriptiva explorativa. Su muestra está conformada por 176 personas, la técnica utilizada es la encuesta y se aplicó como instrumento el cuestionario. Se obtuvo como resultado el $70 \%$ desconoce las obligaciones tributarias, existen conocimientos básicos de las normas tributarias respecto a multas $\mathrm{y}$ sanciones que ponen en riesgo a la empresa y por último los emprendedores del sector textil tienen desconfianza en el pago de impuesto ya que desconocen el destino de su dinero por la corrupción del estado. En conclusión, los comerciantes del mercado no cuentan con una educación tributaria adecuada generando así bajos niveles de cumplimiento de sus impuestos y obteniendo a cambio sanciones y multas que afectan su imagen y crecimiento económico.

De acuerdo a la investigación de Aguirre (2016) el propósito de la fue estudiar la cultura tributaria y su incidencia en el pago de los Impuestos Directos; de diseño descriptivo - exploratoria. Su muestra fue 96 contribuyentes, se aplicó la encuesta y cuestionario para la recolección de datos. Cuyos resultados encontrado señala que la recaudación de impuesto en la ciudad de Ambato no es favorable ya que no cuentan con una cultura tributaria positiva, a pesar de que pagan sus impuestos de forma puntual desconocen los deberes y derechos de sus tributos debido a que tercerizan el servicio, los comerciantes tienen un 
conocimiento básico de los impuestos por ende aún no existe la información necesaria sobre derechos, obligaciones, beneficios y facilidades que las instituciones fiscalizadoras otorga a los contribuyentes. En conclusión, la cultura tributaria si se relaciona positivamente con el cumplimiento de impuestos tributarios en la ciudad de Ambato.

Según Culquicondor (2016) el autor en su investigación tuvo como propuso conocer un programa de orientación tributaria y difusión para promover el cumplimiento tributario voluntario; de diseño descriptivo, no experimental. La muestra está formada por 237 personas propietarios de los predios, la técnica de estudio aplicada es el análisis documentario. Se obtuvo el siguiente resultado de acuerdo al pago de impuesto en el año 2014 y 2015 ha tenido un incremento del $21 \%$ en recaudación, pero esta información no es real ya que el catastro no se encuentra actualizado y por lo tanto no se puede realizar una comparación con lo recaudado y el índice de morosidad, dentro de las causas que generan la evasión de impuestos es por la falta de conocimiento respecto a la aplicación de normas tributarias eimpuestos. En conclusión, los factores que generan una falta de recaudación tributaria son la educación tributario y compromiso de los contribuyentes con el pago de impuestos.

Para Fernández (2020) en su investigación tuvo como propósito conocer el nivel de cultura tributaria en los comerciantes y la prevalencia en la recaudación de impuestos, de tipo descriptiva, correlacional, con diseño no experimental. $\mathrm{Su}$ muestra está conformada por 92 trabajadores del mercado modelo de Chiclayo, se aplicó la encuesta y la observación, el instrumento utilizado para su aplicación es el cuestionario y la lista de observación. Se obtuvo como resultado que el $76 \%$ no se encuentra de acuerdo con las normas tributarias, el $85 \%$ indica que siempre ha tenido problemas con las obligaciones tributarias, el $70 \%$ no deben existir evasión tributaria, el $29 \%$ no cuestiona las normas tributarias a fin de no cometer errores, el $78 \%$ de los comerciantes no emite comprobante de pagos y $69 \%$ desconoce las ventajas tributarias del nuevo RUS. En conclusión, actualmente los comerciantes del mercado modelo no reciben información adecuada generando una deficiencia en su cultura tributaria lo que no les permite disfrutar de los beneficios Recuperadas por entidades supervisora y también porque no tienen el conocimiento necesario de las normas por tal razón evaden impuestos con la única finalidad de no generar pérdidas en la empresa.

Para Capuñay y Silva (2019) su trabajo tuvo como propósito establecer si la cultura tributaria incurre en la evasión de impuestos de los comerciantes, de diseño cuantitativo - pre experimenta. Su muestra está conformada por 66 comerciantes del mercado Moshoqueque; se usó el cuestionario para las dos variables. Se obtuvo como indicador que existe una falta de cultura tributaria en los comerciantes de abarrotes debido a que no asisten a charlas tributarias y por lo tanto desconocen de sus obligaciones tributarias, también los resultados demuestran que los comerciantes evaden impuesto por la falta de emisión de facturas y boletas ya que se siente desalentados por la corrupción que existe en el país. En conclusión, actualmentelos comerciantes evaden sus impuestos debido a la desinformación y a la necesidad de pagar impuesto que para ellos genera una pérdida económica, por lo tanto, las entidades correspondientes tienen que plantear estrategias que permitan capacitar a los comerciantes creando así una cultura tributaria que les permita obtener los beneficios del estado.

De acuerdo a Enrique y García (2019) la investigación tuvo como objetivo formular estrategias que desarrollen una apropiada cultura tributaria para reducir evasión tributaria en los comerciantes de ropa, de tipo descriptivo - cuantitativo. Su muestra está formada por 231 vendedores del mercado modelo, aplico como herramienta de recolección de datos la observación, la encuesta y el análisis documental, aplicando el cuestionario. Los resultados demostraron que el nivel de cultura tributaria 
de los comerciantes es deficiente ya que no tienen el conocimiento necesario sobre las normas tributarias vigentes, esto es debido a la falta de capacitacióncapacitaciones respecto a la importancia que tienen las normas tributarias, el poco interés por parte de la Sunat ente regulador en capacitar a los comerciante o entregar material informativo y la poca información que tienen los comerciantes respecto a la utilidad y beneficios que tienen las micro empresas al pagar sus tributos. En conclusión, las consecuencias que genera tener una cultura tributaria deficientes, por ocasionan altos índices de morosidad en los impuestos ya que los contribuyentes no se sienten motivados en cumplir las normas tributarias y por último se sienten insatisfechos con las obras que realizan en el centro de abastos y que no benefician a comerciantes ni a la sociedad.

\section{Teoría de la función tributaria}

En esta teoría se plantea el derecho tributario, el cual está referido al incremento de la liquidación tributaria, pero que este va a depender de dos factores el primero que se debe realizar una legislación rigurosa a través de una disciplina administrativa a través de los derechos tributarios y el segundo dar autoridad para que a través de la razón las personas con un conjunto de normas tribute (Paredes, 2010).

El sistema tributario es un grupo de procesos que tiene un orden lógico y coherente de tributos que están relacionados con los objetivos de las políticas económicas establecidos a través de principios que se genera a través de los contribuyentes al estado con el objetivo de mantener en el tiempo la calidad de los servicios públicos. Se establece por un conjunto de normas y procedimientos que evalúan y relacionan al contribuyente y acreedor para un mejor funcionamiento (Angelo, 2016).

\section{MÉTODOS Y MATERIALES}

Se aplicó un tipo descriptivo-propositivo, se basa en la descripción de la situación actual de la cultura tributaria y la recaudación de impuestos, se realiza una propuesta con el fin de aumentar la recaudación de impuestos de los comerciantes del mercado Moshoqueque, Chiclayo. La investigación se caracteriza porque parte de la identificación de ciertos problemas de la realidad, a través un diagnóstico descriptivo, luego se realiza una propuesta para dar solución a la problemática (Hurtado, 2010).

Se aplicó un diseño no experimental, de corte transversal, dado que no se hizo manipulación en la variable independiente, sino que solo se describió el nivel de cultura tributaria y el nivel de recaudación de impuestos, tal como se halla en la realidad en un momento determinado. En un diseño no experimental, la variable independiente no es modificada, puesto que son situaciones que ya existen en la realidad, por tal motivo, el investigador solo las describe tal como se hallan es su ambiente natural, y es de corte transversal, porque se analiza en un solo momento determinado (Hernández, Fernández, y Baptista, 2014)
$\mathrm{M}$
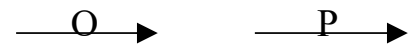
$\mathrm{M}=$ tamaño de la muestra
$\mathrm{O}=$ Observación de la situación actual
$\mathrm{P}=$ Propuesta de cultura tributaria

\section{Población Y Muestra}

Según Hernández et al. (2014), menciona que el grupo de estudio está conformado por todos aquellos individuos que comparten características similares, ubicados en un mismo lugar. El cual estuvo conformado por 76 comerciantes del mercado Moshoqueque, Chiclayo.

La muestra, utilizada fue un muestreo probabilístico simple utilizando la fórmula estadística. En este tipo de muestreo todos los integrantes de la muestra seleccionada han tenido la misma posibilidad de haber sido elegidos (Bernal, 2010). 
Fórmula estadística:

$$
\mathbf{n}=\frac{\mathbf{Z}^{2} \mathbf{p q ~} \mathbf{N}}{\mathbf{E}^{2}(\boldsymbol{N}-\mathbf{1})+\mathbf{Z}^{2} \mathbf{p q}}
$$

$\mathrm{n}=$ Muestra.

$\mathrm{Z}=$ Nivel de confianza (1.96)

$\mathrm{p}=$ Grado de certeza (posibilidad de que

los instrumentos has sido respondidos

adecuadamente, $\mathrm{q}=0.5$ ).

$\mathrm{q}=$ Probabilidad de fracaso (posibilidad de que los instrumentos no has sido respondidos adecuadamente, $\mathrm{q}=0.5$ ).

$\mathrm{E}=$ Margen de error muestral aceptado (5\%).

$\mathrm{N}=$ Población (76 comerciantes del mercado de Moshoqueque).

$$
\mathbf{n}=\frac{(1.96)^{2}(0.5)(0.5)(76)}{(0.05)^{2}(76-1)+(1.96)^{2}(0.5)(0.5)}=64
$$

\section{RESULTADOS}

Objetivo específico 1: Analizar el nivel de cultura tributaria de los comerciantes del mercado Moshoqueque, Chiclayo; se logró luego de la aplicación de una encuesta compuesta por 10 preguntas, del cual se obtuvo los siguientes resultados:

Tabla $N^{\circ} 1$. Análisis del nivel de cultura tributaria de los comerciantes del mercado Moshoqueque

\begin{tabular}{lcc}
\hline \multicolumn{1}{c}{ Categoría } & N & \% \\
\hline Deficiente & 7 & 11 \\
Regular & 33 & 52 \\
Bueno & 22 & 34 \\
Eficiente & 2 & 3 \\
\hline Total & $\mathbf{6 4}$ & $\mathbf{1 0 0}$ \\
\hline
\end{tabular}

Fuente: Resultados de la encuesta.

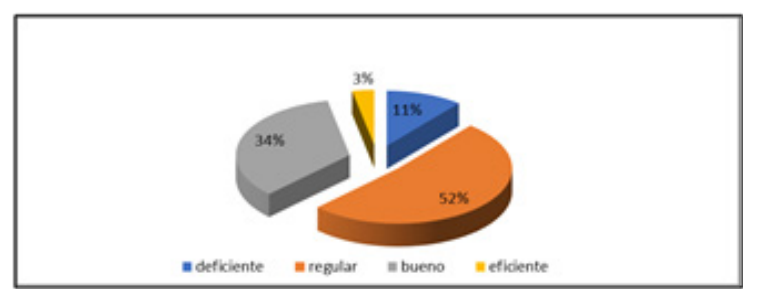

Figura 1. Evaluación del nivel de cultura tributaria de los comerciantes del mercado Moshoqueque
En la tabla 1 y figura 1 se evidencia que el nivel de cultura tributaria de los comerciantes del mercado Moshoqueque se encuentra en un nivel regular según el 52\% de encuestados, debido a que muchos no conocen los requisitos que exigen el régimen tributario al que pertenecen, además que Nunca asisten a charlas de capacitación por parte de la SUNAT.

Objetivo específico 2: Analizar el nivel de recaudación de Impuestos en los comerciantes del mercado Moshoqueque, Chiclayo; se logró luego de la aplicación de una encuesta compuesta por 10 preguntas, del cual se obtuvo los siguientes resultados:

Tabla $\mathrm{N}^{\circ}$ 2. Análisis del nivel de recaudación de Impuestos en los comerciantes del mercado Moshoqueque

\begin{tabular}{lcc}
\hline \multicolumn{1}{c}{ Categoría } & N & \% \\
\hline Deficiente & 9 & 14 \\
Regular & 29 & 45 \\
Bueno & 24 & 38 \\
Eficiente & 2 & 3 \\
\hline Total & $\mathbf{6 4}$ & $\mathbf{1 0 0}$ \\
\hline
\end{tabular}

Fuente: Resultados de la encuesta.

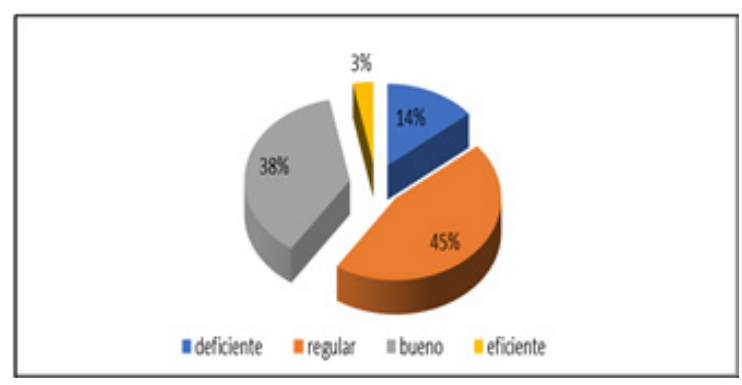

Figura 2. Análisis del nivel de recaudación de Impuestos en los comerciantes del mercado Moshoqueque

En la tabla 2 y la figura 2 se evidencia que la recaudación de Impuestos en los comerciantes del mercado Moshoqueque tiene un nivel regular, según el 45\% de encuestados, debido a que es costumbre que muchos comerciantes no paguen impuestos, o si lo hacen es de manera inadecuada, además omiten comprobantes de pago. 
Objetivo específico 3: Elaborar la estructura de la propuesta de una cultura tributaria para incrementar la recaudación de impuestos de los comerciantes del mercado Moshoqueque, Chiclayo; se logró luego de estructurar la propuesta en base a ciertas estrategias con el fin de incrementar la cultura tributaria en el mercado, las cuales son: Crear un grupo de estudiantes de IX y $\mathrm{X}$ ciclo que brinde charlas de tributación; diseñar y entregar material informativo didáctico simple y claro que brinde la información resaltante sobre normas, obligaciones y beneficios tributarios; realizar un acuerdo con la Sunat y Universidades, para realizar ferias informativas, de consultoría tributaria y difusión de obras públicas por la recaudación de impuestos; realizar charlas de las Universidades con Instituciones Educativas para incentivar la importancia de las obligaciones tributarias en la sociedad; y realizar charlas informativas de tributación.

Objetivo específico 4: Validar la propuesta a juicio de expertos; se logró luego de solicitar el apoyo de un profesional experto, el cual evaluó teniendo en cuenta la siguiente escala: Muy adecuado, bastante adecuado, adecuado, poco adecuado y no adecuado. Donde evaluó aspectos tales como: redacción, estructura de la propuesta, fundamentación teórica, bibliografía $\mathrm{y}$ finalmente, la fundamentación y viabilidad de la propuesta.

El resultado de la evaluación por el experto fue que la propuesta es muy adecuada, respecto a los aspectos antes mencionados, por tal motivo la propuesta se considera confiable y efectiva en caso que se decida aplicar en los comerciantes del mercado Moshoqueque, Chiclayo.

\section{DISCUSIÓN}

Los resultados obtenidos permiten tener un panorama claro acerca de la necesidad de aplicar la propuesta en esta empresa; por lo tanto, se realiza la discusión de resultados, estructurados según el cumplimiento de los objetivos específicos de la investigación contrastando los resultados con investigaciones pasadas y las teorías desarrolladas en el marco teórico.

Respecto al objetivo específico 1: Se pudo evidenciar en los resultados de la tabla 1 que el $30 \%$ de encuestados indicaron que Nunca han identificado a la (SUNAT) como ente administrador, el $16 \%$ contestó que a veces y solo el 11\% respondió que siempre.

Estos resultados coinciden con lo hallado por Bermúdez (2016) en Ecuador, dado que encontró que el $70 \%$ de comerciantes desconoce las obligaciones tributarias, tienen conocimientos básicos de las normas tributarias respecto a multas y sanciones, asimismo los comerciantes tienen desconfianza en el pago de impuesto ya que desconocen el destino de su dinero, se propone el uso de herramientas con el fin de que los comerciantes mejoren su nivel de cultura tributaria; de igual manera se coincide con los resultados hallados por Enrique y García (2019) en Chiclayo, dado que se halló que los comerciantes no tienen conocimiento sobre las normas tributarias vigentes, debido a la falta de capacitación por parte de la SUNAT, asimismo desconocen los beneficios que tienen para las empresas pagar sus impuestos, concluyendo que los comerciantes no se sienten motivados en cumplir las normas tributarias.

Los resultados de la presente investigación respecto al diagnóstico y la descripción de la situación actual de la cultura tributaria, pueden ser utilizados por otras investigaciones que necesiten saber la problemática que acontece en el mercado Moshoqueque, Chiclayo, dónde se recomienda realizar estudios propositivos respecto a la mejora de la cultura tributaria.

Debido a lo anterior se puede concluir que, en el mercado Moshoqueque, se encuentra en una situación de cultura tributaria de nivel bajo, debido a que desconocen los requisitos del régimen tributario al que pertenecen, además los comerciantes en su gran mayoría no asisten a charlas que brinda la SUNAT en temas de cultura 
tributaria, y las orientaciones respecto al pago de tributos que brinda esta entidad es considerada no oportuna para los comerciantes, esta problemática no solo se identifica a nivel local sino también a nivel nacional e internacional.

Respecto al objetivo específico 2: Se pudo evidenciar en los resultados de la tabla 2 se observa que el $25 \%$ de comerciantes opinaron que siempre es común que las personas no paguen impuestos.

Estos resultados coinciden con lo hallado por Aguirre (2016) en Ecuador, donde los comerciantes tercerizan el pago de impuestos, debido a que desconocen los deberes y derechos de sus tributos, conociendo solo aspectos básicos, desconociendo lo beneficios o facilidades que las instituciones fiscalizadoras otorga a los contribuyentes; asimismo se refuerza con lo planteado por Gonzales (2018) el cual menciona que la recaudación tributaria está enmarcada por un conjunto de normas y estrategias que están orientadas a maximizar la captación de los impuestos, tasas y contribuciones, estas facultades permiten a la SUNAT garantizar la recaudación de los tributos.

Los resultados muestran el diagnóstico y la descripción situacional de la recaudación de impuestos, pueden ser utilizados en otras investigaciones que necesiten conocer la realidad problemática del mercado Moshoqueque, y en función a este diagnóstico proponer estrategias para mejorar la recaudación de impuestos.

Debido a lo anterior se puede concluir que, en el mercado Moshoqueque existe una inadecuada recaudación de impuestos, debido a que los comerciantes desconocen el destino de los ingresos por el pago de impuestos, además es común ver que las personas no paguen sus impuestos y que muchos comerciantes omitan comprobantes de pago en sus ventas, finalmente es necesario conocer que esta situación es común tanto a nivel nacional e internacional.

Respecto al objetivo específico 3: Se evidencia que la estructura de la propuesta se hizo en base a ciertas estrategias con el fin de mejorar la cultura tributaria en los comerciantes, las cuales son: Crear un grupo de estudiantes de IX y X ciclo que brinde charlas de tributación; diseñar material informativo didáctico simple y claro que brinde la información resaltante sobre obligaciones y beneficios tributarios; realizar un acuerdo con la Sunat y Universidades, para realizar ferias informativas, de consultoría tributaria y difusión de obras públicas por la recaudación de impuestos; realizar charlas de las Universidades con Instituciones Educativas para incentivar la importancia de las obligaciones tributarias en la sociedad; y realizar charlas informativas de tributación.

Debido a lo anterior se puede concluir que, la estructura de la propuesta de una cultura tributaria tiene como base el diagnóstico realizado, donde se determinó que es necesario que en los comerciantes del mercado Moshoqueque se mejore la educación respecto a temas tributarios, por tal motivo la propuesta está enfocada en estrategias que ayuden a mejorar la cultura tributaria, de tal manera que se genere mayor conciencia en el pago de sus obligaciones tributarias, incrementado de esta manera la recaudación de impuestos.

Respecto al objetivo específico 4: Se pudo evidenciar según el criterio del profesional experto que la propuesta es muy adecuada, es decir es muy efectiva para incrementar la recaudación de impuestos en caso se decida aplicar en los comerciantes del mercado Moshoqueque, debido a que cumple con los criterios de redacción, estructura de la propuesta, fundamentación teórica, bibliografía y finalmente, la fundamentación y viabilidad de la propuesta.

Estos resultados se consolida por Hernández et al. (2014), el cual afirma que la validez es la capacidad que tiene un instrumento para generalizar resultados o medir verdaderamente lo que se está buscando.

Los resultados respecto a la validación de la propuesta por juicio de expertos, pueden ser 
utilizados en otras investigaciones que necesiten conocer criterios para considerar que una propuesta es adecuada para ser aplicada en una empresa. Se recomienda que en futuras investigaciones se considere la metodología de como una propuesta ha sido considerada adecuada antes de ser aplicada.

Debido a lo anterior se puede concluir que, una propuesta de una cultura tributaria debe ser validada por profesionales expertos, con la finalidad de evaluar su aplicabilidad en los contribuyentes, garantizando su efectividad para incrementar la recaudación de impuestos.

\section{CONCLUSIONES}

Al analizar el nivel de cultura tributaria; se concluye que existe un nivel regular, a causa de factores como: desconocimiento del régimen tributario al que pertenecen, los comerciantes no asisten a charlas ofrecidas por la SUNAT, y debido a que no se brinda una información oportuna a los comerciantes por parte de la SUNAT.

Al analizar el nivel de recaudación de Impuestos, se concluye que existe un nivel regular, debido a que es habitual que muchos comerciantes no paguen impuestos, o lo realizan de manera inadecuada, omitiendo comprobantes de pago para evadir impuestos, asimismo existen comerciantes que desconocen el proceso de recaudación tributaria. Al elaborar la estructura de la propuesta de una cultura tributaria para incrementar la recaudación de impuestos de los comerciantes del mercado Moshoqueque, Chiclayo; se concluye que la propuesta permitirá incrementar la recaudación de impuestos, dado que se han establecido estrategias enfocados en mejorar la cultura tributaria de los comerciantes, mediante las charlas informativas.

Al realizar la validación de la propuesta por juicio de expertos; se concluye que la propuesta es muy adecuada según criterio del experto, teniendo en cuenta la redacción, estructura de la propuesta, fundamentación teórica y viabilidad; por lo tanto, se considera aplicable en los comerciantes del mercado Moshoqueque con el fin de incrementar la recaudación de impuestos.

\section{REFERENCIAS BIBLIOGRÁFICAS}

Aguirre, A. (2016). La cultura tributaria y su relación con el pago de los impuestos directos de la ciudad de Ambato, provincia de Tungurahua, 2015. (Tesis de pregrado, Universidad Técnica de Ambato, Ambato, Ecuador). Obtenido de https:// tinyurl.com/ybeuffcv

Angelo, C. (2016). La evasión y su incidencia en la recaudación tributaria en la provincia de Huancayo. (Tesis de pregrado, Universidad Nacional del Centro del Perú, Huancayo, Perú). Obtenido de https://is.gd/fPHQq7

Bermúdez, G. (2016). La cultura tributaria y el pago de impuestos de los expendedores de ropa y afines del Mercado Mayorista cantón Ambato. (Tesis de pregrado, Universidad Técnica de Ambato, Ambato, Ecuador). Obtenido de https:// tinyurl.com/ya4dswx9

Bernal,C.(2010). Metodología dela investigación: administración, economía, humanidades y ciencias sociales (3 ed.). Colombia: Pearson Educación. doi:ISBN: 978-958-699-128-5

Capuñay, J., \& Silva, J. (2019). Cultura tributaria y su incidencia en la evasión de impuestos en los comerciantes de abarrotes del mercado Moshoqueque-Lambayeque 2017. (Tesis de pregrado, Universidad Señor de Sipán, Pimentel, Perú). Obtenido de https://tinyurl.com/y79l38ks

Centro de Gestión Tributaria de Chiclayo. (28 de Setiembre de 2018). CGT Chiclayo Realizó Campaña de Educación Tributaria en Instituciones Educativas. Centro de Gestión Tributaria de Chiclayo. Obtenido de https:// tinyurl.com/y7xp76h2

Culquicondor, B. (2016). Factores que influyen el cumplimiento tributario voluntario de la 
recaudación del impuesto predial, Distrito de Santiago de Cao- Ascope 2015. (Tesis de pregrado, Universidad César Vallejo, Trujillo, Perú). Obtenido de https://tinyurl.com/y76brpwk

Enrique, J., \& García, M. (2019). La cultura tributaria para disminuir la evasión tributaria en los comerciantes de ropa ubicados en los alrededores del mercado modelo de Chiclayo-2017. (Tesis de pregrado, Universidad Señor de Sipán, Pimentel, Perú). Obtenido de https://inyurl.com/yaczo72n

Gonzales, C. (2018). Estrategias para incrementar la recaudación tributaria en la municipalidad provincial de Ferreñafe. (Tesis de Maestría). Universidad Cesar Vallejo. Ferreñafe. Obtenido de https://tinyurl.com/ydgdul8r

Hernández, R., Fernández, C., \& Baptista, P. (2014). Metodología de la investigación (6 ed.). México: Mcgraw-Hill/Interamericana Editores, S.A. Obtenido de https://is.gd/iREcBj

Hurtado, J. (2010). Metodología de la investigación guía para la comprensión holística de la ciencia (4 ed.). Caracas: Quiros Ediciones. Obtenido de https://is.gd/ele0Xx

Paredes, C. (2010). El Poder Tributario del Estado: ¿Nuestros Bolsillos a la Deriva? . Derecho Tributario , 241-256. Obtenido de https://is.gd/ SX8hba 